\title{
Evaluation of the Effectiveness of Rotational Conjunctival Flap Compared to Free Autografting for Ocular Surface Reconstruction after Surgical Removal of Primary Pterygium
}

\author{
Abu Bakr Mohammad Farid Abul Naga, Ahmed Gomaa EL Mahdi, Sultan Hussien Ali Hasan* \\ Department of Ophthalmology, Faculty of Medicine, Al-Azhar University, Cairo, Egypt \\ *Corresponding author: Sultan Hussien Ali Hasan, Mobile: (+20)01066385522, E-Mail: sultan.hussien2@ gmail.com
}

\begin{abstract}
Background: pterygium is a fibrovasular lesion of the ocular surface that can display an aggressive clinical behavior and occasionally, threatens the vision. Although there is no consensus on its pathogenesis, recent evidence suggests that is a prolifrative rather than degenerative condition, strongly correlated with exposure to ultraviolet radiation of solar light. Objictive: the purpose of this study was to compare the surgical outcomes when using the technique of conjunctival autograft versus conjunctival rotational flap for reconstruction of ocular surface following primary pterygium excision. Patients and Methds: this study included 20 eyes of 20 patients with primary pterygia attending at the National Eye Center, Rod El Afarag Hospital. Operated eyes were divided into 2 groups, 10 eyes each Group A: included 10 eyes managed by pterygium excision followed by ocular surface reconstruction using rotational conjunctival flap technique. Group B: included 10 eyes managed by pterygium excision followed by ocular surface reconstruction using free conjunctival autograft technique. Results: The better result wase in cases treated by conjunctival autograft group with $10 \%$ recuurence while the higher recurrence rate $20 \%$ was seen in cases by rotational conjunctival flap group. Other minor complications were found such as flap retraction in 2 cases of group, buttonhole of the graft in one case in group B, flap edema was in lower incidence than graft edema and no suture granuloma happened in both groups.
\end{abstract}

Conclusion: free conjunctival autograft and rotational conjuctiavl flap showed comparable results, in reducing recuurence rate; they are safe and effective methods of pterygium surgery that produces only few complications.

Keywords: Conjunctival, Autografting for Ocular Surface Reconstruction, Pterygium

\section{INTRODUCTION}

Pterygium is a fibrovascular proliferation that grows at the inter-palpebral conjunctival area, it can occur on either side of the cornea, but the nasal limbus is much more commonly involved ${ }^{(\mathbf{1})}$.

Its growth can cause also irregular astigmatism, corneal scarring, restriction of ocular motility and chronic ocular surface inflammation ${ }^{(\mathbf{1})}$.

Pterygium may be active (red and thickened) and grow over months to years, or inactive (white and flat) and remain stable for years ${ }^{(2)}$.

Although the pathogenesis of a pterygium remains an enigma, several risk factors were reported for the occurrence of pterygium such as ultra-violet (UV) light, repeated micro-trauma by dust, chronic conjunctival inflammation, genetic predisposition and ocular dryness have all been reported to be involved in the development of pterygium, indicating a multifactorial pathogenesis ${ }^{(\mathbf{1})}$. Existing reports indicate that sunlight exposure is the main factor in pterygium occurrence by inducing growth factor production or chronic inflammation or Deoxyribonucleic acid (DNA) damage ${ }^{(3)}$.Multiple procedures have been advocated in the treatment of pterygium. These range from simple surgical excision, the 'bare sclera technique', it is an easy and common procedure used to manage pterygium ${ }^{(4)}$. However, this procedure is associated with a recurrence rate as high as $80 \%$. Application of MMC (MMC), beta-radiation, amniotic membrane and conjunctival transplant have been used in conjunction with simple surgical excision to reduce recurrence ${ }^{(5,6)}$.
Conjunctival tissue, either as rotation flap or free autograft has been used in conjunction with simple surgical excision to reduce the recurrence and avoid the side effects associated with the use of MMC ${ }^{(7)}$.

\section{AIM OF THE STUDY}

The purpose of this study was to compare the surgical outcomes when using the technique of conjunctival autograft versus conjunctival rotational flap for reconstruction of ocular surface following primary pterygium excision.

\section{PATIENTS AND METHODS}

This prospective interventional comparative study included a total of 20 eyes of 20 patients with primary pterygia that warrant surgical removal, attending at the National Eye Center, Rod El Afarag Hospital. Written informed consent from all the subjects were obtained. This study was conducted between (Mention the duration e.g. January 2016, and December 2017). Approval of the ethical committee was obtained. Operated eyes were divided into 2 groups of 10 eyes each:

- Group A: using the rotational conjunctival flap technique.

- Group B: using free conjunctival autograft technique.

\section{All patients were subjected to the following: Pre-operative assessment \\ - History taking and complete ocular examination. \\ - Ocular examination including refraction and assessment of best corrected visual acuity, slit}


lamp biomicroscopy examination and digital anterior segment photography.

Grading of pterygium for all cases by using Anbesse et al. ${ }^{(8)}$ system:

- Grade 1: extends less than $2 \mathrm{~mm}$ onto the cornea. A deposit of iron (Stocker line) may be seen in the corneal epithelium anterior to the advancing head of the pterygium.

- Grade 2: involves up to $4 \mathrm{~mm}$ of the cornea and may be primary or recurrent following surgery.

- Grade 3: encroaches onto more than $4 \mathrm{~mm}$ of the cornea and involves the visual axis.

Laboratory assessment of plasma glucose levels, bleeding time and clotting time to detect and exclude patients with abnormal results, which may impair healing.

\section{Operative procedure:}

All procedures were done with standard ophthalmologic sterile preparation, using operating microscope. Topical surface anesthesia in the form of surface anesthesia in the form of benoxate Hydrochloride $0.4 \%$ was instilled first then pribulbar anesthesia with $2 \%$ lignocaine and $0.5 \%$ bupivacaine in 1: 1 ratio was injected.

The eye was draped, lid speculum was inserted then the size of the pterygium was measured from the limbus to the pterygium apex using a surgical caliper.

The pterygium head was separated from the cornea by blunt and sharp dissection and the conjunctiva with underlying Tenon's capsule was excised, down to the bare sclera. Only the thickened portion of conjunctiva and the immediate adjacent and subjacent Tenon's capsule showing tortuous vasculature were excised. Large hemorrhage stopped with direct compression while cautery was avoided as possible.

In group A, the flap was made by $\mathrm{U}$ shaped incision in the upper bulbar conjunctiva to inferonasal edge is made adjacent to the wound to form a tongue of conjunctiva, the flap was thinly dissected from Tenon's capsule then was rotated in the place of bare sclera. The flap was placed at least $1 \mathrm{~mm}$ of bare sclera adjacent to the cornea-scleral then sutured by $8 / 0$ polyglycolic acid interrupted sutures (fig. 1).

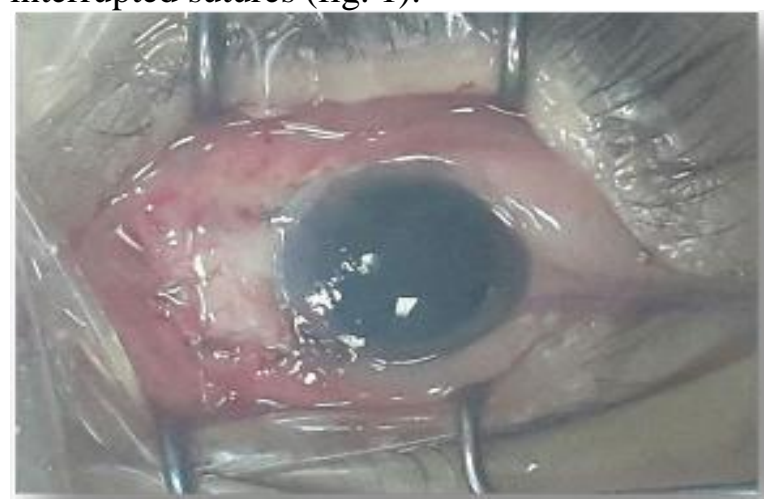

Fig. (1): Intra operative photo of patient after fixation of CRF by 0.8 vicryl suture.

In group B, the graft was approximately 1-

$2 \mathrm{~mm}$ greater than bare sclera size and was taken from Superior temporal bulbar conjunctiva. Conjunctival graft was dissected from the Tenon for obtaining the thinnest possible conjunctiva then placed on the bare sclera. Care was taken to ensure proper orientation of the graft with the epithelial side up and limbal side toward the limbus, then the graft was sutured by $8 / 0$ polyglycolic acid interrupted sutures. (fig. 2)

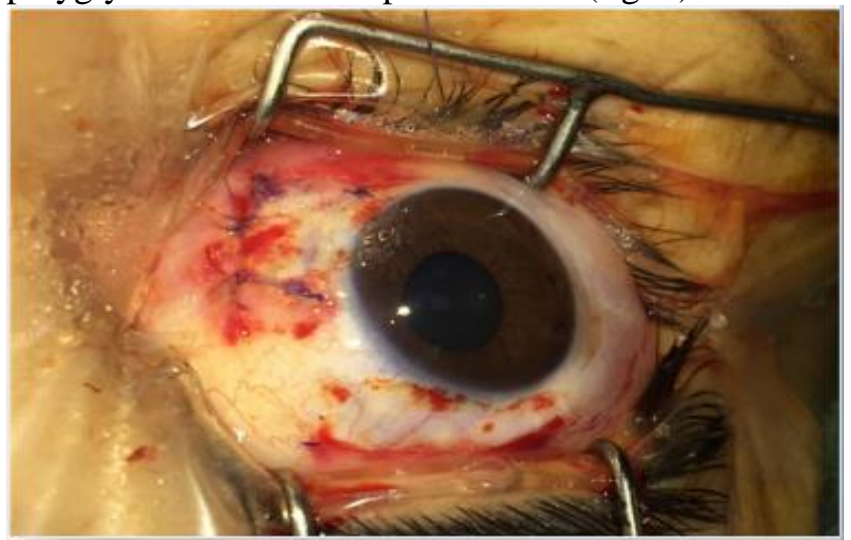

Fig. (2): Intraoperative view of patient after fixation of CAG by vicryl 0.8 .

Topical antibiotic and steroid combination eye drops were administered 4 times a day for 10 a days and tapered over the next week. Lubricant eye drops were administered 3 times a day for 1 month. The patients were followed up post operatively after 24 hours, 1 weeks, 6 weeks and then at 3 to 6 months.

\section{Post-Operative assessment}

1. Slit lamp examination and digital anterior segment photography was done to assess for complications and pterygium recurrence.

2. The subjective symptoms of patients including stitching pain, foreign body sensation and lacrimation were questioned at every follow up.

3. Recurrence of the pterygium was defined as the presence of fibrovascular tissue regrowth extending onto clear cornea within the follow-up period using caliper.

4. Refraction was performed in the 6th week.

Primary outcomes: To determine the incidence of dislocation or retraction in the graft or the flap and the rate of pterygium recurrence in both techniques.

Secondary outcome: To assess the intra operative complication such as Graft or flap buttonhole and postoperative complications such as sub conjunctival hemorrhage, edema and granuloma formation.

\section{Statistical analysis}

Recorded data were analyzed using the statistical package for social sciences, version 20.0 (SPSS Inc., Chicago, Illinois, USA). Quantitative data were expressed as mean \pm standard deviation (SD). Qualitative data were expressed as frequency and percentage.

\section{The following tests were done:}

- Independent-samples t-test of significance was used when comparing between two means.

- Chi-square $\left(x^{2}\right)$ test of significance was used in order to compare proportions between two qualitative parameters. 
- The confidence interval was set to $95 \%$ and the margin of error accepted was set to 5\%. The p-value was considered significant as the following:

- Probability (P-value)

- P-value $<0.05$ was considered significant.

- P-value <0.001 was considered as highly significant.

- P-value >0.05 was considered insignificant.

\section{RESULTS}

The study included 20 eyes of 20 patients; 12 female and 8 male with mean age 46.40 ys in group A and 46.50 ys in group B. all cases of The pterygium were primary in both groups, the complain of patients and degree of encroachment shown in Table (1).

Table (1): Comparison between rotational flap group and auto graft group regarding demographic data, complain and degree of encroachment of the studied cases

\begin{tabular}{|c|c|c|c|c|c|c|}
\hline & Rotational flap group & Auto graft group & \multirow{2}{*}{ Test value } & \multirow{2}{*}{ P-value } & \multirow{2}{*}{ Sig. } \\
\hline & & No. $=10$ & No. $=10$ & & & \\
\hline Gender & $\begin{array}{l}\text { Females } \\
\text { Males }\end{array}$ & $\begin{array}{l}6(60.0 \%) \\
4(40.0 \%) \\
\end{array}$ & $\begin{array}{l}6(60.0 \%) \\
4(40.0 \%)\end{array}$ & 0.000 & 1.000 & NS \\
\hline Age (years) & $\begin{array}{l}\text { Mean } \pm \text { SD } \\
\text { Range }\end{array}$ & $\begin{array}{l}46.40 \pm 10.00 \\
29-60\end{array}$ & $\begin{array}{l}46.50 \pm 7.43 \\
32-54\end{array}$ & 0.025 & 0.980 & NS \\
\hline Complain & $\begin{array}{l}\text { Cosmetic } \\
\text { Visual }\end{array}$ & $\begin{array}{l}3(30.0 \%) \\
7(70.0 \%) \\
\end{array}$ & $\begin{array}{l}4(40.0 \%) \\
6(60.0 \%) \\
\end{array}$ & 0.220 & 0.639 & NS \\
\hline $\begin{array}{l}\text { Degree of } \\
\text { encroachment }\end{array}$ & $\begin{array}{l}\text { Mild }(<2 \mathrm{~mm}) \\
\text { Moderate }(2-3 \mathrm{~mm}) \\
\text { Severe }(>3 \mathrm{~mm})\end{array}$ & $\begin{array}{l}2(20.0 \%) \\
4(40.0 \%) \\
4(40.0 \%)\end{array}$ & $\begin{array}{l}1(10.0 \%) \\
6(60.0 \%) \\
3(30.0 \%)\end{array}$ & 0.876 & 0.645 & NS \\
\hline
\end{tabular}

$\mathrm{P}>0.05$ : Non significant (NS); P < 0.05: Significant (S); P < 0.01: Highly significant (HS).*: Chi-square test; $\bullet:$ Independent t-test

\section{Operative data of the studied groups: \\ Intra operative:}

No intra operative graft or flap buttonhole was noted in group A but 2 patients in group B. Table (2) shows the difference between group A and group B. However, There was a statistically non significant difference in the graft or flap buttonhole occurrence between the two groups with $(\mathrm{P}$ value $=0.136)$.

Table (2): Comparison between rotational flap and auto graft group group regarding graft or flap buttonhole

\begin{tabular}{|l|l|l|l|l|l|l|l|}
\hline \multirow{2}{*}{$\begin{array}{l}\text { Graf or flap } \\
\text { button hole }\end{array}$} & \multicolumn{2}{|l|}{ Rotational flap group } & \multicolumn{2}{|l|}{ Auto graft group } & \multirow{2}{*}{ Test value } & \multirow{2}{*}{ P-value } & \multirow{2}{*}{ Sig. } \\
\cline { 2 - 6 } & No. & \% & No. & \% & & \\
\hline No & 10 & $100.0 \%$ & 8 & $80.0 \%$ & \multirow{2}{*}{$2.222 *$} & \multirow{2}{*}{0.136} & NS \\
\hline Yes & 0 & $0.0 \%$ & 2 & $20.0 \%$ & & & \\
\hline Total & 10 & $100.0 \%$ & 10 & $100.0 \%$ & & \\
\hline
\end{tabular}

*: Chi-square test

Postoperative data of the studied groups:

The patients were asked to subjectively note the following complications stitching pain, excessive lacrimation and foreign body sensation on post operative first day post operarive, week post operative,first month and three to six months.

\section{Postoperative stitching pain represented in Table (3)}

Table (3): Shows the difference in postoperative stitching pain between group A and group B

\begin{tabular}{|c|c|c|c|c|c|c|c|c|}
\hline & \multicolumn{2}{|c|}{ Group A } & \multicolumn{2}{|c|}{ Group B } & \multirow{2}{*}{$\begin{array}{l}\text { Test } \\
\text { value* }^{*}\end{array}$} & \multirow{2}{*}{ P-value } & \multirow{2}{*}{ Sig. } \\
\hline & & No. & $\%$ & No. & $\%$ & & & \\
\hline \multirow{2}{*}{$\begin{array}{l}\text { Postop. } \\
\text { Stitching pain }\end{array}$} & No & 0 & $0.0 \%$ & 0 & $0.0 \%$ & \multirow{2}{*}{0.000} & \multirow{2}{*}{1.000} & \multirow{2}{*}{ NS } \\
\hline & Yes & 10 & $100.0 \%$ & 10 & $100.0 \%$ & & & \\
\hline \multirow{2}{*}{1 day } & No & 0 & $0.0 \%$ & 0 & $0.0 \%$ & \multirow{2}{*}{0.000} & \multirow{2}{*}{1.000} & \multirow{2}{*}{ NS } \\
\hline & Yes & 10 & $100.0 \%$ & 10 & $100.0 \%$ & & & \\
\hline \multirow{2}{*}{7 day } & No & 2 & $20.0 \%$ & 2 & $20.0 \%$ & \multirow{2}{*}{0.000} & \multirow{2}{*}{1.000} & \multirow{2}{*}{ NS } \\
\hline & Yes & 8 & $80.0 \%$ & 8 & $80.0 \%$ & & & \\
\hline \multirow{2}{*}{1 months } & No & 7 & $70.0 \%$ & 8 & $80.0 \%$ & \multirow{2}{*}{0.267} & \multirow{2}{*}{0.606} & \multirow{2}{*}{ NS } \\
\hline & Yes & 3 & $30.0 \%$ & 2 & $20.0 \%$ & & & \\
\hline \multirow{2}{*}{3 months } & No & 10 & $100.0 \%$ & 10 & $100.0 \%$ & \multirow{2}{*}{0.000} & \multirow{2}{*}{1.000} & \multirow{2}{*}{ NS } \\
\hline & Yes & 0 & $0.0 \%$ & 0 & $0.0 \%$ & & & \\
\hline \multirow{2}{*}{6 months } & No & 10 & $100.0 \%$ & 10 & $100.0 \%$ & \multirow{2}{*}{0.000} & \multirow{2}{*}{1.000} & \multirow{2}{*}{ NS } \\
\hline & Yes & 0 & $0.0 \%$ & 0 & $0.0 \%$ & & & \\
\hline
\end{tabular}

*: Chi-square test 
All patients experienced stitching pain in first day post operatively but, after one week, there was a statistically non significant difference in postoperative stitching pain between the two groups, with decrease in stitching pain noted by patients in group B more than group A. Pain sensation declined markedly by the end of the first month and was not detected by the end of follow up.P value (1.000) .

\section{Post operative excessive lacrimation:}

Post-operative excessive lacrimation noted by patients is summarized in Table (4).

Table (4): Shows difference in postoperative lacrimation in post operative first day post operarive, week post operative,first month and three to six months.

\begin{tabular}{|c|c|c|c|c|c|c|c|c|}
\hline & \multicolumn{2}{|c|}{ Rotational flap group } & \multicolumn{2}{|c|}{ Auto graft group } & \multirow{2}{*}{$\begin{array}{c}\text { Test } \\
\text { value* }\end{array}$} & \multirow{2}{*}{ P-value } & \multirow{2}{*}{ Sig } \\
\hline & & No. & $\%$ & No. & $\%$ & & & \\
\hline $\begin{array}{l}\text { Postop. excessive } \\
\text { lacrimation }\end{array}$ & $\begin{array}{l}\text { No } \\
\text { Yes }\end{array}$ & $\begin{array}{l}0 \\
10 \\
\end{array}$ & $\begin{array}{l}0.0 \% \\
100.0 \%\end{array}$ & $\begin{array}{l}1 \\
9 \\
\end{array}$ & $\begin{array}{l}10.0 \% \\
90.0 \%\end{array}$ & 1.053 & 0.305 & NS \\
\hline 1 day & $\begin{array}{l}\text { No } \\
\text { Yes }\end{array}$ & $\begin{array}{l}0 \\
10\end{array}$ & $\begin{array}{l}0.0 \% \\
100.0 \%\end{array}$ & $\begin{array}{l}3 \\
7\end{array}$ & $\begin{array}{l}30.0 \% \\
70.0 \%\end{array}$ & 3.529 & 0.060 & NS \\
\hline 7 day & $\begin{array}{l}\text { No } \\
\text { Yes }\end{array}$ & $\begin{array}{l}7 \\
3 \\
\end{array}$ & $\begin{array}{l}70.0 \% \\
30.0 \% \\
\end{array}$ & $\begin{array}{l}8 \\
2 \\
\end{array}$ & $\begin{array}{l}80.0 \% \\
20 . \% \\
\end{array}$ & 0.837 & 0.360 & NS \\
\hline 1 months & $\begin{array}{l}\text { No } \\
\text { Yes }\end{array}$ & $\begin{array}{l}10 \\
0\end{array}$ & $\begin{array}{l}100.0 \% \\
0.0 \%\end{array}$ & $\begin{array}{l}10 \\
0\end{array}$ & $\begin{array}{l}100.0 \% \\
0.0 \%\end{array}$ & 0.000 & 1.000 & NS \\
\hline 3 months & $\begin{array}{l}\text { No } \\
\text { Yes }\end{array}$ & $\begin{array}{l}10 \\
0\end{array}$ & $\begin{array}{l}100.0 \% \\
0.0 \%\end{array}$ & $\begin{array}{l}10 \\
0\end{array}$ & $\begin{array}{l}100.0 \% \\
0.0 \%\end{array}$ & 0.000 & 1.000 & NS \\
\hline 6 months & $\begin{array}{l}\text { No } \\
\text { Yes }\end{array}$ & $\frac{10}{0}$ & $\begin{array}{l}100.0 \% \\
0.0 \%\end{array}$ & $\frac{10}{0}$ & $\begin{array}{l}100.0 \% \\
0.0 \%\end{array}$ & 0.000 & 1.000 & NS \\
\hline
\end{tabular}

*: Chi-square test

There was no statistically insignificant difference in postoperative lacrimation between the two groups on from first day post operative through out the follow up period

\section{Post operative foreign body sensation:}

Post-operative foreign body sensation was noted by patients in both groups as represented in Table (5).

Table (5): Shows difference in postoperative foreign body (F.B.) sensation between two groups

\begin{tabular}{|c|c|c|c|c|c|c|c|c|}
\hline & \multicolumn{2}{|c|}{ Rotational flap group } & \multicolumn{2}{|c|}{ Auto graft group } & \multirow{2}{*}{$\begin{array}{l}\text { Test } \\
\text { value* }\end{array}$} & \multirow{2}{*}{ P-value } & \multirow{2}{*}{ Sig. } \\
\hline & & No. & $\%$ & No. & $\%$ & & & \\
\hline $\begin{array}{l}\text { Postop. foreign } \\
\text { body sensation }\end{array}$ & $\begin{array}{l}\text { No } \\
\text { Yes }\end{array}$ & $\begin{array}{l}0 \\
10\end{array}$ & $\begin{array}{l}0.0 \% \\
100.0 \%\end{array}$ & $\begin{array}{l}0 \\
10\end{array}$ & $\begin{array}{l}0.0 \% \\
100.0 \%\end{array}$ & 0.000 & 1.000 & NS \\
\hline 1 day & $\begin{array}{l}\text { No } \\
\text { Yes }\end{array}$ & $\begin{array}{l}0 \\
10 \\
\end{array}$ & $\begin{array}{l}0.0 \% \\
100.0 \%\end{array}$ & $\begin{array}{l}0 \\
10 \\
\end{array}$ & $\begin{array}{l}0.0 \% \\
100.0 \% \\
\end{array}$ & 0.000 & 1.000 & NS \\
\hline 7 day & $\begin{array}{l}\text { No } \\
\text { Yes }\end{array}$ & $\begin{array}{l}6 \\
4\end{array}$ & $\begin{array}{l}60.0 \% \\
40.0 \%\end{array}$ & $\begin{array}{l}7 \\
3\end{array}$ & $\begin{array}{l}70.0 \% \\
30.0 \%\end{array}$ & 0.220 & 0.639 & NS \\
\hline 1 months & $\begin{array}{l}\text { No } \\
\text { Yes }\end{array}$ & $\begin{array}{l}7 \\
3 \\
\end{array}$ & $\begin{array}{l}70.0 \% \\
30.0 \% \\
\end{array}$ & $\begin{array}{l}9 \\
1 \\
\end{array}$ & $\begin{array}{l}90.0 \% \\
10.0 \% \\
\end{array}$ & 1.25 & 0.263 & NS \\
\hline 3 months & $\begin{array}{l}\text { No } \\
\text { Yes }\end{array}$ & $\begin{array}{l}9 \\
1\end{array}$ & $\begin{array}{l}90.0 \% \\
10.0 \%\end{array}$ & $\begin{array}{l}10 \\
0\end{array}$ & $\begin{array}{l}100.0 \% \\
0.0 \%\end{array}$ & 1.053 & 0.304 & NS \\
\hline 6 months & $\begin{array}{l}\text { No } \\
\text { Yes }\end{array}$ & $\begin{array}{l}10 \\
0\end{array}$ & $\begin{array}{l}100.0 \% \\
0.0 \%\end{array}$ & $\begin{array}{l}10 \\
0\end{array}$ & $\begin{array}{l}100.0 \% \\
0.0 \%\end{array}$ & 0.000 & 1.000 & NS \\
\hline
\end{tabular}

*: Chi-square test

There was a statistically non significant difference in postoperative foreign body (F.B.) sensation between both groups from day 1 , day 7 ( $p$ value $=\mathbf{1 . 0 0}$ ) and still statistically non significant in the first month ( $\mathrm{p}$ value $=0.639$ ) and follow up 3 months there was statistically also non significant difference with ( $p$ value $=0.304$ ) with F.B sensation much greater in group A, then on 6 months this complain disappeared in both groups (table 5).

\section{Visual acuity and astigmatism:}

Visual acuity was measured preoperatively and one month post-operatively for each patient in both groups. The distribution of different visual acuities in both groups is shown in Table (6), improvement of vision was noted in 8 patients in group A versus 6 patients in group B and the rest o f patients were the same visual acuity. However, the difference between 2 groups was statistically insignificant in preoperative $(\mathrm{P}$ value $=0.785)$ and post operative $(\mathrm{P}$ value $=1.000)$, but each group show significant improvement of visual acuity on log mar chart (Table 6). 
Table (6): Preoperative and post-operative visual acuity in both groups

\begin{tabular}{|c|c|c|c|c|c|c|}
\hline & $\begin{array}{l}\text { Rotational } \\
\text { flap group }\end{array}$ & \begin{tabular}{|l} 
Auto graft \\
group
\end{tabular} & \multirow[t]{2}{*}{ Test value } & \multirow[t]{2}{*}{ P-value } & \multirow[t]{2}{*}{ Sig. } \\
\hline & & No. $=\mathbf{1 0}$ & No. $=\mathbf{1 0}$ & & & \\
\hline Preop. visual acuity & $\begin{array}{l}\text { Mean } \pm \text { SD } \\
\text { Range }\end{array}$ & $\begin{array}{l}0.55 \pm 0.17 \\
0.3-0.8\end{array}$ & $\begin{array}{l}0.56 \pm 0.15 \\
0.3-0.8\end{array}$ & $0.277 \bullet$ & 0.785 & NS \\
\hline Postop. visual acuity & $\begin{array}{l}\text { Mean } \pm \text { SD } \\
\text { Range }\end{array}$ & $\begin{array}{l}0.65 \pm 0.12 \\
0.5-0.8\end{array}$ & \begin{tabular}{|l|}
$0.65 \pm 0.14$ \\
$0.4-0.8$
\end{tabular} & $0.000 \bullet$ & 1.000 & NS \\
\hline Paired t-test & $\begin{array}{l}\mathrm{T} \\
\mathrm{p} \text {-value }\end{array}$ & \begin{tabular}{|l|}
3.714 \\
$0.010(\mathbf{S})$
\end{tabular} & \begin{tabular}{|l|}
2.862 \\
$0.019(\mathbf{S})$
\end{tabular} & & & \\
\hline
\end{tabular}

$\bullet:$ Independent t-test

\section{Post-operative complications:}

The recurrence was considered when fibrovascular growth had occurred at the site of previously excised pterygium crossing the limbus and extending onto the cornea.

Each patient in both groups was examined for post-operative complications such as graft or flap loss or retraction, graft or flap injection, recurrence, granuloma formation and sub conjunctival hemorrhage on first day, week, one month and three months post operatively, Table (7).

Table (7): Post operative complications

\begin{tabular}{|c|c|c|c|c|c|c|c|c|}
\hline & & \multicolumn{2}{|c|}{$\begin{array}{l}\text { Rotational flap } \\
\text { group }\end{array}$} & \multicolumn{2}{|c|}{$\begin{array}{l}\text { Auto graft } \\
\text { group }\end{array}$} & \multirow[t]{2}{*}{ Test value* } & \multirow[t]{2}{*}{ P-value } & \multirow[t]{2}{*}{ Sig. } \\
\hline & & No. & $\%$ & No. & $\%$ & & & \\
\hline Postop. Recurrence & $\begin{array}{l}\text { No } \\
\text { Yes }\end{array}$ & $\begin{array}{l}8 \\
2 \\
\end{array}$ & $\begin{array}{l}80.0 \% \\
20.0 \% \\
\end{array}$ & $\begin{array}{l}9 \\
1 \\
\end{array}$ & $\begin{array}{l}90.0 \% \\
10.0 \% \\
\end{array}$ & 0.392 & 0.531 & NS \\
\hline $\begin{array}{l}\text { Postop. flab } \\
\text { graft loss }\end{array}$ & $\begin{array}{l}\text { No } \\
\text { Yes }\end{array}$ & $\begin{array}{l}10 \\
0 \\
\end{array}$ & $\begin{array}{l}100.0 \% \\
0.0 \%\end{array}$ & $\begin{array}{l}10 \\
0 \\
\end{array}$ & $\begin{array}{l}100.0 \% \\
0.0 \%\end{array}$ & 0.000 & 1.000 & NS \\
\hline $\begin{array}{l}\text { Postop. flab } \\
\text { graft retract }\end{array}$ & $\begin{array}{l}\text { No } \\
\text { Yes }\end{array}$ & $\begin{array}{l}7 \\
3\end{array}$ & $\begin{array}{l}70.0 \% \\
30.0 \%\end{array}$ & $\begin{array}{l}10 \\
0\end{array}$ & $\begin{array}{l}100.0 \% \\
0.0 \%\end{array}$ & 3.529 & 0.060 & NS \\
\hline \multirow[b]{2}{*}{ Postop. Granuloma } & No & 10 & $100.0 \%$ & 10 & $100.0 \%$ & \multirow[b]{2}{*}{0.000} & \multirow[b]{2}{*}{1.000} & \multirow[b]{2}{*}{ NS } \\
\hline & $\begin{array}{l}\text { Yes } \\
\text { Yes }\end{array}$ & $\begin{array}{l}0 \\
0\end{array}$ & $\begin{array}{l}0.0 \% \\
0.0 \%\end{array}$ & $\begin{array}{l}0 \\
0\end{array}$ & $\begin{array}{l}0.0 \% \\
0.0 \%\end{array}$ & & & \\
\hline
\end{tabular}

*: Chi-square test

Post- operative recurrence: One cases developed recurrence of pterygium after three months in group A (20\%) and one case in group B $(20 \%)$, The difference between the two groups was statistically insignificant (p value $=0.329)$ (Table 7).

Post-operative graft or flap loss There was no reported graft or flap loss in either of the studied groups $(0.0 \%)$ (Table 7$)$.

Post-operative graft or flap retaction: Three patients had flap retraction in group A (30.0\%) one of them in day 1 and 2 of them in day 7 of the follow up with no graft retraction in group B but it was statistically non significant difference (P-value 0.060 ) between the both groups (Table 7).

Post-operative granuloma: There was no reported granuloma in either of the studied groups (Table 7).

\section{Post-operative sub conjunctival hemorrhage:}

There was a statistically non significant difference in occurrence of postoperative subconjunctival hemorrhage between the two groups on day land day 7, being higher in the rotational flap group and complete resolution at 1 month follow up in both groups as shown in (Table 8) 
Table (8): Shows postoperative subconjunctival hemorrhage in both groups

\begin{tabular}{|c|c|c|c|c|c|c|c|c|}
\hline & & \multicolumn{2}{|c|}{$\begin{array}{l}\text { Rotational flap } \\
\text { group }\end{array}$} & \multicolumn{2}{|c|}{$\begin{array}{l}\text { Auto graft } \\
\text { group }\end{array}$} & \multirow{2}{*}{$\begin{array}{l}\text { Test } \\
\text { value* }\end{array}$} & \multirow[t]{2}{*}{ P-value } & \multirow[t]{2}{*}{ Sig. } \\
\hline & & No. & $\%$ & No. & $\%$ & & & \\
\hline $\begin{array}{l}\text { Postop. sub conjunctival } \\
\text { hemorrhage }\end{array}$ & $\begin{array}{l}\text { No } \\
\text { Yes }\end{array}$ & $\begin{array}{l}7 \\
3\end{array}$ & $\begin{array}{l}70.0 \% \\
30.0 \%\end{array}$ & $\begin{array}{l}3 \\
7\end{array}$ & $\begin{array}{l}30.0 \% \\
70.0 \%\end{array}$ & 3.200 & 0.074 & NS \\
\hline 1 day & $\begin{array}{l}\text { No } \\
\text { Yes }\end{array}$ & $\begin{array}{l}7 \\
3 \\
\end{array}$ & $\begin{array}{l}70.0 \% \\
30.0 \% \\
\end{array}$ & $\begin{array}{l}3 \\
7 \\
\end{array}$ & $\begin{array}{l}30.0 \% \\
70.0 \% \\
\end{array}$ & 3.200 & 0.074 & NS \\
\hline 7 day & $\begin{array}{l}\text { No } \\
\text { Yes }\end{array}$ & $\begin{array}{l}7 \\
3 \\
\end{array}$ & $\begin{array}{l}70.0 \% \\
30.0 \% \\
\end{array}$ & $\begin{array}{l}3 \\
7 \\
\end{array}$ & $\begin{array}{l}30.0 \% \\
70.0 \% \\
\end{array}$ & 3.200 & 0.074 & NS \\
\hline 1 months & $\begin{array}{l}\text { No } \\
\text { Yes }\end{array}$ & $\begin{array}{l}10 \\
0\end{array}$ & $\begin{array}{l}100.0 \% \\
0.0 \%\end{array}$ & $\begin{array}{l}10 \\
0\end{array}$ & $\begin{array}{l}100.0 \% \\
0.0 \%\end{array}$ & 0.000 & 1.000 & NS \\
\hline
\end{tabular}

*: Chi-square test

\section{Post-operative graft or flap edema within one weak.}

In the first week follow up we noticed flap edema in only 2 eyes in group a (20\%) and 4 cases of graft edema in group b (40\%) then all resolved within the first month follow up.

Table (9): Postoperative graft edema

\begin{tabular}{|l|l|l|l|l|l|l|l|l|}
\hline \multicolumn{2}{|c|}{} & \multicolumn{2}{l|}{$\begin{array}{l}\text { Rotational flap } \\
\text { group }\end{array}$} & \multicolumn{2}{l|}{$\begin{array}{l}\text { Auto graft } \\
\text { group }\end{array}$} & \multirow{2}{*}{$\begin{array}{l}\text { Test } \\
\text { value* }\end{array}$} & \multirow{2}{*}{ P-value } & \multirow{2}{*}{ Sig. } \\
\cline { 3 - 9 } & No. & $\%$ & No. & $\%$ & & \\
\hline $\begin{array}{l}\text { Postop. graft edema } \\
\text { (chemosis) within } \\
\text { one weak }\end{array}$ & No & 8 & $80.0 \%$ & 6 & $60.0 \%$ & & & \\
\hline
\end{tabular}

*: Chi-square test

\section{CASE PRESNTATION}

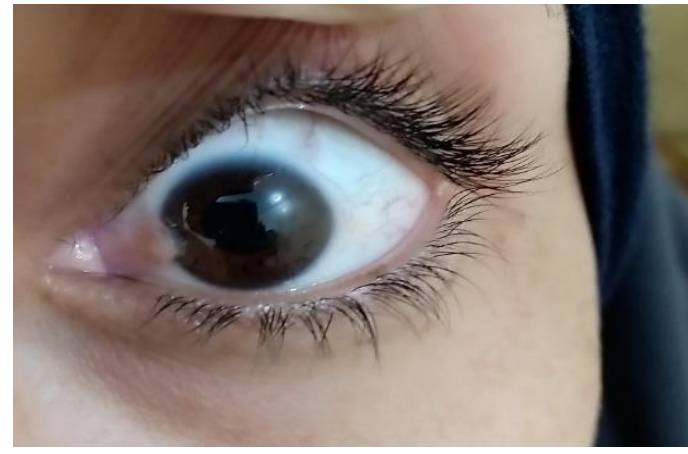

Preoperative

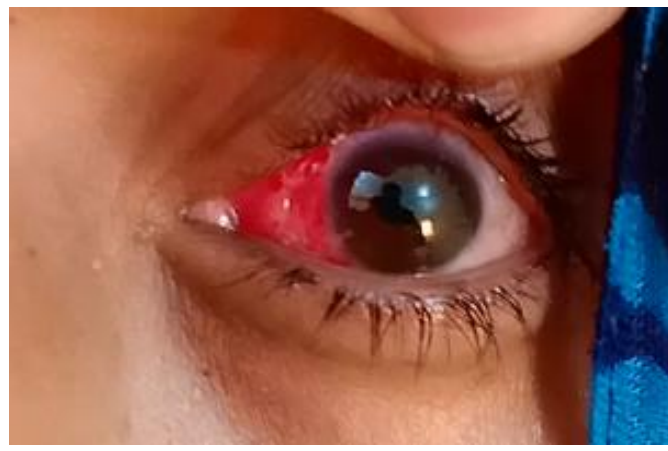

After 7 day

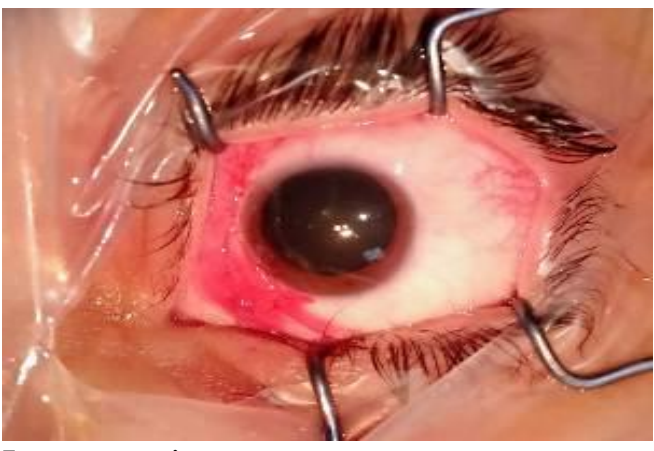

Intraoperative

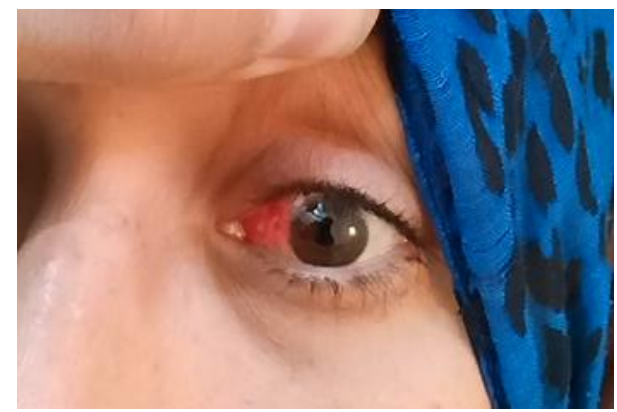

After 1 months 


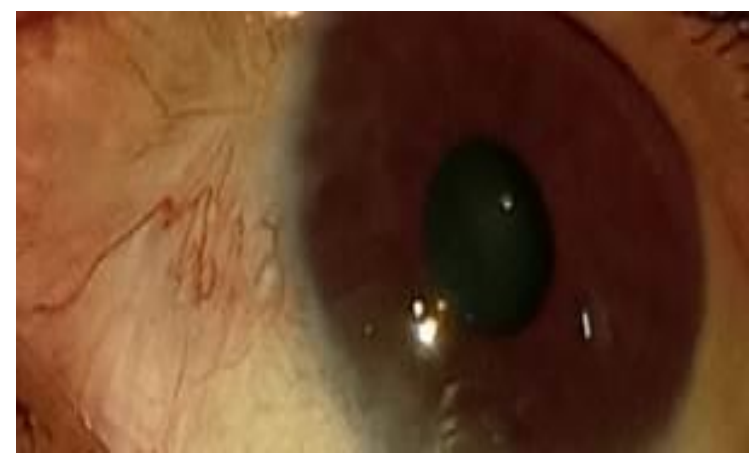

After 6 months

Fig. (1): Patients of group A.

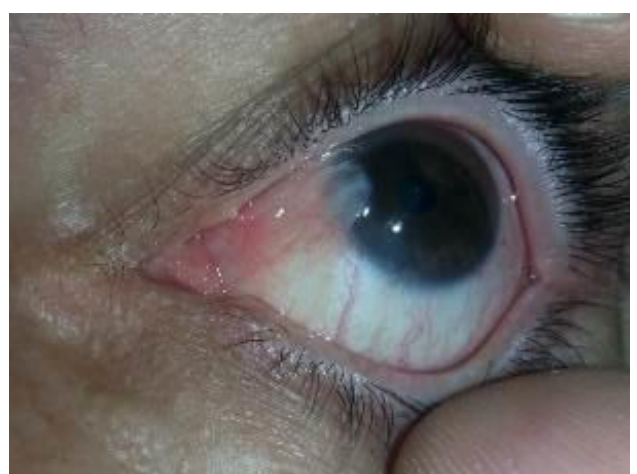

Preoperative

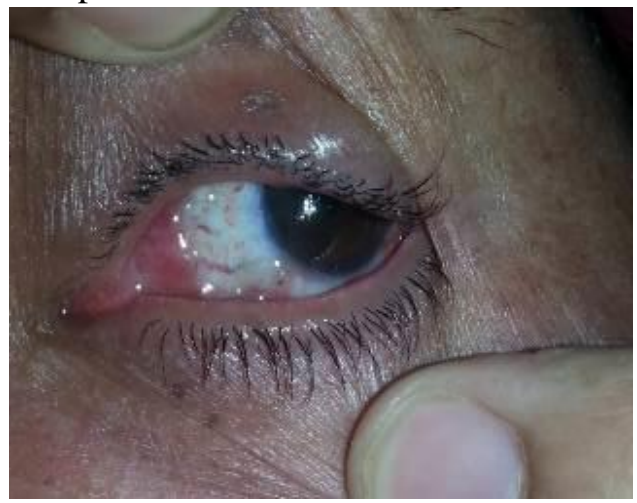

After 1 month

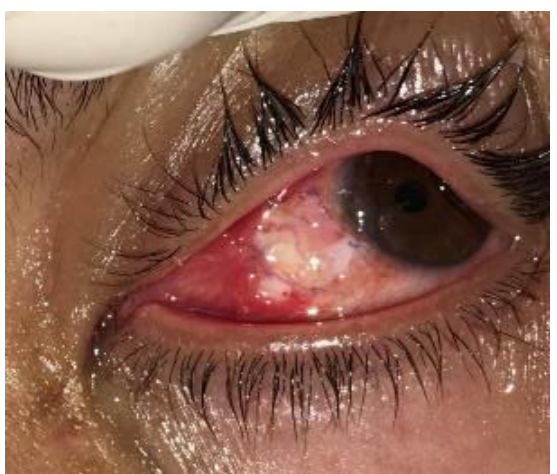

After one week

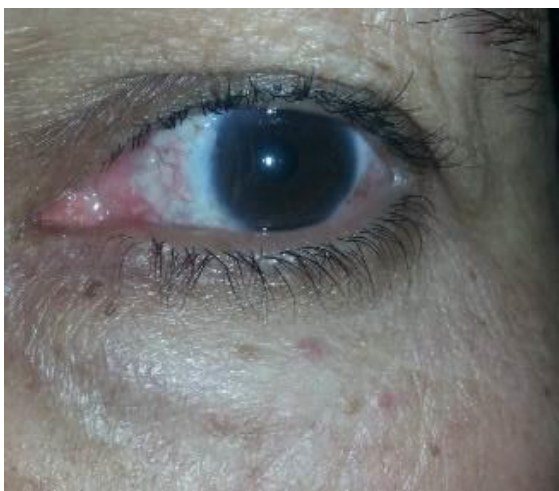

After 6 months

Fig. (4): Patients of group B.

\section{DISCUSSION}

Group A study: In our study conjunctival rotational flap transplantation technique has been applied on 10 cases (Group A) out of 20 cases $(50 \%)$, whose age ranged from 29 to 60 years with mean $46.40 \pm 10.00$, there were 4 males (40\%) and 6 females $(60 \%)$, where 7 cases (70\%) complaining from visual disturbance and 3 cases (30\%) complaining from cosmetic annoyance. All cases had unilateral and primary pterygium, severity of pterygium was graded from mild pterygium (2 cases, $20 \%$ ), moderate pterygium (4 cases, $40 \%$ ) and severe pterygium ( 4 cases, $40 \%$ ).

Group B study: Limbal conjunctival auto graft transplantation technique has been applied on 10 cases out of 20 cases (50\%), whose age ranged from 32 to 54 years with mean 46.50 , there were 4 males $(40 \%)$ and 6 females $(60 \%)$, where 6 cases $(60 \%)$ complaining from visual disturbance and 4 cases (40\%) complaining from cosmetic annoyance. All cases had unilateral and primary pterygium, severity of pterygium was graded from mild pterygium (1 case, 10\%), moderate pterygium (6 cases, 60\%) and severe pterygium (3 cases, $30 \%)$.

The follow-up period was (3-6 months) for both groups and the recurrence was considered when fibrovascular growth had occurred at the site of previously excised pterygium crossing the limbus and extending onto the cornea.

\section{Regarding postoperative complication}

1. In group A study:

There was recurrence in 2 cases $(\mathbf{2 0 \%})$, flap edema detected in 2 eyes (20\%)in the first weak then complete disappeared after that and no reported cases of 
granuloma formation except for mild congestion and hemorrhage of the conjunctival flap but all of them disappeared within one month.

No reported cases of flap buttonhole or graft loss except for 1 case of flap retraction found in the first post-operative day due to rubbing of eyes causing disruption of the one stitch.

\section{In group b study:}

There were recurrence of one case $(10 \%)$ as follow: 39 years, male patient, complaining of visual disturbance due to presence of moderate pterygium (2$3 \mathrm{~mm}$ corneal encroachment) which is unilateral and primary in origin graft edema in 4 cases (40\%), 2 cases with buttonhole (20\%), this happened during separation of the tenon from the conjunctival graft, no reported cases of post-operative graft retraction or graft loss, no reported cases of granuloma formation except for mild congestion and sub conjunctival hemorrhage in 7 cases (70\%) during the first week post poreative but all of them disappeared within one month.

Regarding the patient complaining of ocular pain, foreign body sensation and excessive lacrimation in both groups

1. Regarding post operative stitching pain: All patients experienced stitching pain in first day post operatively but, after one week started to disappear, there was a statistically non-significant difference in postoperative stitching pain between the two groups, with decrease in stitching pain noted by patients in group B $(20 \%)$ more than group $\mathrm{A}(30 \%)$ in the first month. Pain sensation declined markedly by the end of the first month and was not detected by the end of follow up, $\mathrm{P}$ value (1.000), in both groups.

\section{Regarding post-operative foreign body sensation:} All cases in the rotational conjunctival flap experienced foreign body sensation but 9 eyes in autograft experienced foreign body sensation in the first postoperative day then disappeared this complain in both groups though the follow up peroid, statistically non significance between both groups with F.B sensation much greater in group A.

3. Regarding post-operative excessive lacrimation: Most of cases had excessive lacrimation but all of this improved during of follow up, and the rotational flap show more excessive lacrimation and more longer than autograft cases, the There was no statistically insignificant difference in postoperative lacrimation between the two groups on day 1 ( $\mathrm{p}$ value $=\mathbf{0 . 3 0 5}$ ) and highly significant difference on day 7 ( $\mathrm{p}$ value $=\mathbf{0 . 0 0 1}$ ) and significant difference in one month ( $p$ value $=\mathbf{0 . 0 1 9}$ ) follow up then This complaint disappeared in both groups afterwards.

Excessive lacrimation within the first weak was 2 cases $(20 \%)$ in first month (autograft).

Regarding the visual outcome and the astigmatic changes in both groups
1. The visual acuity: In our study, in group $A, 8$ patients $(80 \%)$ showed improvement of visual acuity and 6 patients $(60 \%)$ in group B. and best corrected visual acuity showed significant improvement $0.54 \pm$ 0.17 to $0.65 \pm 0.12$ in rotational flap and significant improvement in mean Log MAR visual acuity from $0.56 \pm 0.15$ to $0.65 \pm 0.14$. in auto graft, the difference between 2 groups was statistically insignificant in preoperative $(\mathrm{P}$ value $=0.785)$ and post-operative $(\mathrm{P}$ value $=1.000$ ),

2. Astigmatic changes: In our study there was decrease in astigmatism post operatively in both groups but this difference was not statistically significant in the postoperative astigmatism between the two groups ( $\mathrm{p}$ value $=0.682$ )

In 2014, Akhter et al. ${ }^{(5)}$ applied study about rotational conjunctival flap on 31 eyes (18 males \& 13 females) with primary pterygium, they reported different complications with different percentages as graft edema in 3 eyes $(9.3 \%)$ but in our study 2 eyes $(20 \%)$ it was, granuloma formation in one eye (3.1\%) but no cases in our study and they reported recurrence in 2 eyes out of 26 eyes (7.69\%) but we reported two cases (20\%) our higher perecentage was due to small number of the operated eyes

In 2013, Aslan et al. ${ }^{(9)}$ applied study of Wide Conjunctival Flap on 23 patients (13 males and 10 females), mean age $48.21 \pm 15.13$ years, postoperative recurrence was seen in three cases $(13 \%)$ at $13.2 \pm 3.8$ months of follow-up period and our recuurence was 2 eyes $(20 \%)$. Flap edema was not seen but, in our study, there were 2 cases $(20 \%)^{(9)}$.

Park et al. ${ }^{(10)}$ applied study that include Effect of transverse conjunctival advancement flap surgery for primary pterygium as a new technique it was Retrospective, noncomparative, interventional caseseries analysis on 63 eyes of 63 patients with primary pterygium were treated with transverse conjunctival advancement flap technique, the patients were followed up for more than 6 months. The mean age of patients was $64.9 \pm 8.7$ years (range $43-85$ years), and the mean follow-up period was $9.8 \pm 3.9$ months (range 6-18 months). During the follow-up period, recurrence of the pterygium occurred in $1(1.5 \%)$ of the 63 cases, with recurrence only in the conjunctiva. There was no occurrence of serious complications such as formation of granuloma or conjunctival infection an it was near of our results except for lower incidence of recurrence than our study and may this because of the different technique of the conjunctival flap

Hong et al. ${ }^{(11)}$ applied study on 59 eyes in 59 patients with primary pterygium who were treated with pterygium excision and an inferior conjunctival transposition flap Patients. The mean patient age was 55.6 (ranging from 34 to 74 ) years. The mean follow-up period was 24.9 (ranging from 12 to 46 ) months. During the follow-up period, the pterygium recurred in one $(1.7 \%)$ of the 59 eyes. There were no severe complications except for mild congestion and 
hemorrhage of the conjunctival flap. But all of them disappeared within one month, and there were no serious complications so it has near results and low recurrence rate more than our study may this because small number of our study group and good technique to prevent recurrence.

In 2007, Baig et al. ${ }^{(12)}$ applied study of Sliding Conjunctival Flap Technique on 48 patients. Patients were followed up for 1 year post-surgically at regular intervals Results: It was found that at the end of 1 year, $3(6.25 \%)$ developed recurrence.

In 2012, Chen et al. ${ }^{(13)}$ applied study To evaluate the long-term efficacy in eyes that had been treated with rotation conjunctival flap for pterygium, Totally 66 cases (71 eyes) of pterygium which had been operated by this way were followed up for an average of 3.85 years, the congestion situation in the location of the canthus wrinkles fold as well as eye movements to determine the effect of surgery. And they found during the follow-up of 14 months to 9 years, 58 eyes were cured, 12 eyes were basically cured, 1 eye recurred, surgical recurrence rate was $1.4 \%$.

Kim et al. (14) applied study of anchored conjunctival rotation flap techniques on (35 eyes) of primary pterygium and were followed up postoperatively for a minimum period of 18 months. The recurrence rate was $8.6 \%$ in the anchored conjunctival rotational flap group However, the occurrence of flap or graft edema (14.3\%). No granuloma formation was observed in either group.

Our patients' conditions were close to corresponding conditions of Salman and Mansour ${ }^{(15)}$ who applied free limbal conjunctival auto graft on 20 eyes with pterygium: 16 males (80\%) and 4 females (20\%), whose age ranged from 30 to 50 years old). Also, Mejía et al. ${ }^{(16)}$ applied free limbal conjunctival auto graft on 24 eyes with primary pterygia: the mean age of the study group was 42.5 years (range, 23-75).

On the other hand, the present study patients' conditions were inconsistent with Pulte et al. ${ }^{(17)}$ who applied limbal conjunctival auto graft on 70 eyes, 62 eyes with primary pterygium and 8 eyes with recurrent pterygium, and Mashhour and Al Fayez ${ }^{(18)}$ who applied free limbal conjunctival auto graft on 43 cases of primary and recurrent pterygia: 42 males and 1 female, as well.

Through follow-up period (3-6 months), the noticed complications are as following: excessive lacrimation within the first weak was 2 cases $(20 \%)$ in first month and graft edema in 4 cases (40\%), recurrence in one case (10\%) as follow: 39 years, male patient, complaining of visual disturbance due to presence of moderate pterygium (2-3 $\mathrm{mm}$ corneal encroachment) which is unilateral and primary in origin.

Regarding the recurrence These results are consistent with Salman and Mansour ${ }^{(15)}$ who reported a recurrence in 2 eyes out of 20 eyes (10\%) after follow up period of 6 to 15 months, Mejía et al. ${ }^{(16)}$ who reported a recurrence in one case out of 24 cases $(4.1 \%)$ after follow up period of 3 to12 months, Pulte et al. ${ }^{(17)}$, who reported a recurrence in 2 cases out of 70 cases $(2.7 \%)$, Mashhour and Al Fayez (18) who reported no recurrences.

Yousuf ${ }^{(19)}$ in his study of the role of pterygium excision in pterygium induced astigmatism which included 50 eyes by using autograft technique, found that mean refractive astigmatism improved from $-4.3 \pm 1.88 \mathrm{D}$ preoperatively to $-2.11 \pm 1.9 \mathrm{D}$ postoperatively which is also following the general agreement between researchers and BCVA significantly improved only in $40 \%$ of cases and remained the same in the remaining $60 \%$ which is close to our findings.

In the study of Shelke et al. ${ }^{(20)}$ using autograft technique, significant improvement in the visual acuity after pterygium excision seen in grade II and grade III pterygia $(\mathrm{P}<0.001)$ but in grade IV, the visual acuity was improved but it was not statistically significant because of remnant corneal opacity in the visual axis. In our study we excluded any case with central corneal opacity which caused that difference in results.

Altan-Yaycioglu et al. ${ }^{(21)}$ studied 240 eyes that underwent pterygium excision by 5 different types of surgeries: conjunctival autograft with sutures $(\mathrm{N}=115)$ or fibrin glue $(\mathrm{N}=53)$, conjunctival rotational flap $(\mathrm{N}=47)$, or amniotic membrane transplantation with either suture $(\mathrm{N}=15)$ or with glue $(\mathrm{N}=10)$. Following surgery, astigmatic values decreased from $3.47 \pm 2.50 \mathrm{D}$ to $1.29 \pm$ $1.07 \mathrm{D}(\mathrm{P}<0.001$, paired $\mathrm{t}$ test $)$. The changes in astigmatism was significantly related to the preoperative size of the pterygium $(\rho=3.464, P=0.005)$. The postoperative astigmatism correlated with preoperative astigmatism $(\rho=0.351, P<0.001$, Spearman correlation analysis), finally it was found that Pterygium results in high corneal astigmatism, which decreases to an acceptable level following excision. According to our study, the type of grafting as CAG, CRF does not have a significant effect on the change in astigmatism degree.

For visual outcome in our study, the results were parallel the most of the previous discussed studies. In group A, 8 patients (80\%) showed improvement of visual acuity and 6 patients (60\%) in group B. and best corrected visual acuity showed significant improvement in mean Log MAR visual acuity from $0.56 \pm 0.15$ to $0.65 \pm 0.14$. in auto graft and highly significant improvement $0.54 \pm 0.17$ to $0.65 \pm 0.12$ in rotational flap, the difference between 2 groups was statistically insignificant in preoperative $(\mathrm{P}$ value $=0.785)$ and postoperative $(\mathrm{P}$ value $=1.000)$, almost studies regarding visual acuity and refraction was done on auto graft techniques so we didn't have comparable studies for our rotational flap group except for Altan-Yaycioglu $\boldsymbol{e t}$ al. (21).

In the current study, astigmatism was measured preoperatively and one month postoperatively for each patient in both groups and it significantly reduced in both groups. The degree of astigmatism was reduced in (6)patients in group A, compared to (7) patients in group B.There was no 
statistically significant difference in astigmatism pre operative or post operatively between the two groups.

Astigmatism was not analyzed in most of the other similar studies, except in 2013, Altan-Yaycioglu et al. (21).

\section{CONCLUSION AND RECOMMENDATIONS}

Free conjunctival auto graft and rotational conjuctiavl flap showed comparable results, in reducing recuurence rate, they are safe and effective methods of pterygium surgery that produces only few complications. They produce anatomic barrier and so effectively lower recuurence rate, cosmotic and surgical results ewre encouraging.

It is recommended to do further researches on large number of cases with longer follow up period and more analysis of post-operative signs such as graft edema, corneal scar and objective scoring system for post-operative symptoms. It is also recommend performing corneal topography before and after pterygium removal for more assessment of astigmatism changes.

\section{REFERENCES}

1. Detorakis ET and Spandidos DA (2009): Pathogenetic mechanisms and treatment options for ophthalmic pterygium: trends and perspectives. Int J Mol Med., 23(4): 439-47.

2. Satish K, Gopinath G, Akshatha S et al. (2015): Conjunctival autographing in recurrent pterygium: a retrospective study. International Journal of Scientific Study, 3(3):321-327.

3. Anguria P, Kitinya J, Ntuli S et al. (2014): The role of heredity in pterygium development. Int J Ophthalmol., 7(3): 563-73.

4. Alpay A, Uğurbaş SH and Erdoğan B (2009): Comparing techniques for pterygium surgery. Clini Ophthalmol., 3: 69-74.

5. Akhter W, Tayyab A, Kausar A et al. (2014): Reducing postoperative pterygium recurrence: comparison of free conjunctival auto-graft and conjunctival rotation flap techniques. J Coll Phys Surg Pak., 24: 740-4.

6. Ozgurhan EB, Agca A, Kara N et al. (2013): Topical application of bevacizumab as an adjunct to recurrent pterygium surgery. Cornea, 32(6): 835-8.
7. Bhatia J, Varghese M, Narayanadas B et al. (2013): Cut-and-place technique of pterygium excision with autograft without using sutures or glue: Our experience. Oman J Ophthalmol., 10(2): 81-6.

8. Anbesse DH, Kassa T, Kefyalew B et al. (2017): Prevalence and associated factors of pterygium among adults living in Gondar city, Northwest Ethiopia. PLoS One, 12(3): e0174450.

9. Aslan L, Aslankurt M, Aksoy A et al. (2013): Comparison of wide conjunctival flap and conjunctival autografting techniques in pterygium surgery. J Ophthalmol., 209401. doi: 10.1155/2013/209401.

10. Park J, Son Y and Suh E (2016): Effect of transverse conjunctival advancement flap surgery for primary pterygium. Can J Ophthalmol., 51(6): 401-407.

11. Hong SM, Ko SJ and Kim SD (2009): Effect of inferior conjunctival transposition flap surgery for primary pterygium. J Korean Ophthalmol Soc., 50: 1774-9.

12. Baig MA, Asghar S and Ishaq M (2007): Sliding conjunctival flap in the surgical management of Pterygium: a better approach. Pak Armed Forces Med J., 57(3): 206-11.

13. Chen SY, Liu G and Li YL (2012): Long-term efficacy of rotation conjunctival flap for pterygium. Guoji Yanke Zazhi (Int Eye Sci), 12(9): 1779-80.

14. Kim SH, Oh JH, Do JR, Chuck RS et al. (2013): A comparison of anchored conjunctival rotation flap and conjunctival autograft techniques in pterygium surgery. Cornea, 32(12): 1578-81

15. Salman AG and Mansour DE (2011): The recurrence of pterygium after different modalities of surgical treatment. Saudi Journal of Ophthalmology, 25(4): 411-5.

16. Mejía LF, Sánchez JG and Escobar H (2005): Management of primary pterygia using free conjunctival and limbal-conjunctival autografts without antimetabolites. Cornea, 24(8): 972-5.

17. Pulte P, Heiligenhaus A, Koch J et al. (1998): Long-term results of autologous conjunctiva-limbus transplantation in pterygium. Klin Monbl Augenheilkd, 213(1):9-14.

18. Mashhour F and Al Fayez V (2002): Limbal versus Conjunctival Auto graft Transplantation for Advanced and Recurrent Pterygium. Ophthalmology, 109: 1752-5.

19. Yousuf $M$ (2005): Role of pterygium excision in pterygium induced astigmatism. JK-Practitioner, 12(2): 91-2.

20. Shelke E, Kawalkar U, Wankar R et al. (2014): Effect of pterygium excision on pterygium induced astigmatism and visual acuity. Int $\mathbf{J}$ Adv Health Sci., 40(9): 24-4.

21. Altan-Yaycioglu R, Kucukerdonmez C, Karalezli A et al. (2013): Astigmatic changes following pterygium removal: Comparison of 5 different methods. Indian J Ophthalmol., 61: 104-8. 